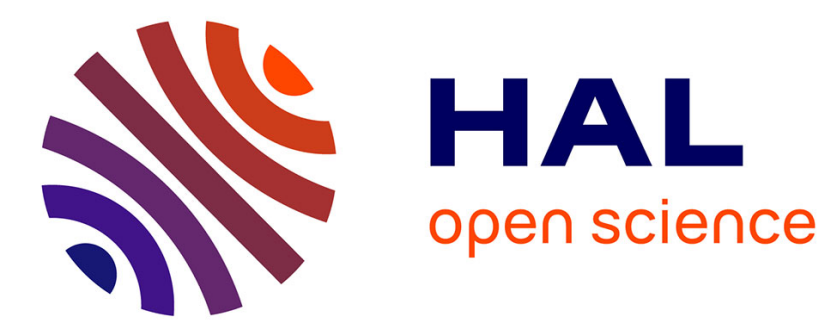

\title{
Warp-based Helical Implicit Primitives
}

Cédric Zanni, Evelyne Hubert, Marie-Paule Cani

\section{To cite this version:}

Cédric Zanni, Evelyne Hubert, Marie-Paule Cani. Warp-based Helical Implicit Primitives. Computers and Graphics, 2011, Computers \& Graphics, 35 (3), pp.517-523. 10.1016/j.cag.2011.03.027 . inria00605339

\section{HAL Id: inria-00605339 \\ https://hal.inria.fr/inria-00605339}

Submitted on 1 Jul 2011

HAL is a multi-disciplinary open access archive for the deposit and dissemination of scientific research documents, whether they are published or not. The documents may come from teaching and research institutions in France or abroad, or from public or private research centers.
L'archive ouverte pluridisciplinaire HAL, est destinée au dépôt et à la diffusion de documents scientifiques de niveau recherche, publiés ou non, émanant des établissements d'enseignement et de recherche français ou étrangers, des laboratoires publics ou privés. 


\title{
Warp-based Helical Implicit Primitives
}

\author{
C. Zanni ${ }^{1,2}$, E. Hubert ${ }^{3}$, M.-P. Cani ${ }^{1,2}$ \\ ${ }^{1}$ Laboratoire Jean-Kutzmann, University of Grenoble, France \\ ${ }^{2}$ INRIA Grenoble - Rhône-Alpes, France, ${ }^{3}$ INRIA Sophia Antipolis - Méditérrannée, France
}

\begin{abstract}
Implicit modeling with skeleton-based primitives has been limited up to now to planar skeletons elements, since no closed-form solution was found for convolution along more complex curves. We show that warping techniques can be adapted to efficiently generate convolution-like implicit primitives of varying radius along helices, a useful 3D skeleton found in a number of natural shapes. Depending on a single parameter of the helix, we warp it onto an arc of circle or onto a line segment. For those latter skeletons closed form convolutions are known for entire families of kernels. The new warps introduced preserve the circular shape of the normal cross section to the primitive.
\end{abstract}

Categories and Subject Descriptors (according to ACM CCS): Computer Graphics [I.3.5]: Computational Geometry and Object Modeling-

\section{Introduction}

Implicit surfaces, defined as $f(x, y, z)=c$, where $f$ is a smooth function and $c$ a given iso-value, provide an easy way to design organic-looking shapes. They can be used in a constructive modeling framework where smooth shapes are progressively blended into more complex ones by simply summing their field functions. Among them, convolution surfaces define $f$ as the integral of a kernel function $K$ along a skeleton $\mathcal{S}$ of lower dimension (a set of points, curves and surfaces). This skeleton, along which we can assign a fluctuating weight, provides an intuitive control of a shape with varying thickness. Thanks to the additivity of the integral operator, different partition of the skeleton leave the convolution function unchanged. Convolution surfaces thus naturally lend themselves to skeleton subdivision techniques for refinement. However, convolution surfaces require a closed form expressions of their field functions to achieve reasonable computing time. So far, this has been found for planar skeletons such as line segments, arcs of circle, quadratic curves or triangles. For this reason, 3D skeleton curves are typically approximated by a series of planar curve segments. Depending on the number of those planar primitives, either the computational efficiency or the visual quality of the shape is impaired.

In this paper we introduce for the first time an analytical implicit primitive around the simplest proper space curve, the circular helix; it has constant curvature and

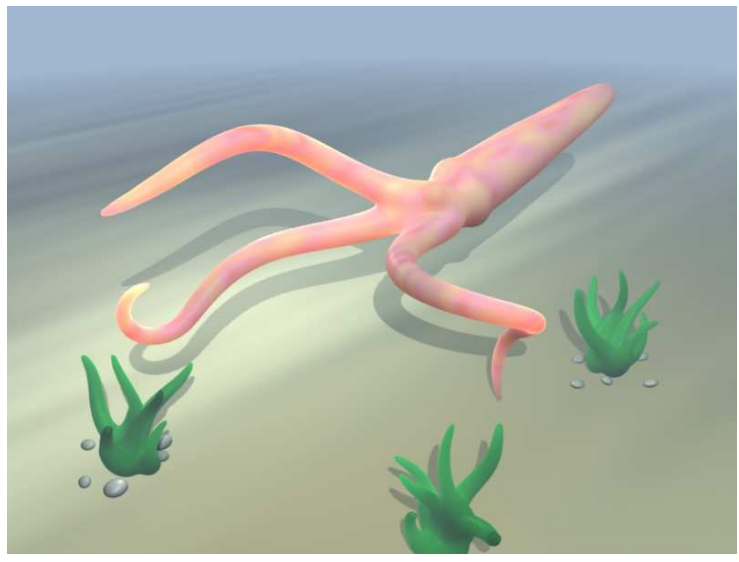

Figure 1: Implicit modeling of a squid. Each tentacle is made of two helical primitives.

constant torsion. It is a promising high level primitive for tessellating arbitrary 3D curves and can be used by itself for modeling realistically a wide range of organic shapes, from hair locks [BAC $\left.{ }^{*} 06\right]$ to natural branching structures such as trees or antlers, see Figures 1 and 16.

\subsection{Related Work}

Convolution surfaces, defined as the level sets of a scalar field obtained by integrating a kernel function along a skeleton, were introduced to provide bulgefree implicit modeling over complex skeletal elements 
such as graphs of branching curves [BS91]. The numerical evaluation used there for the parametric integrals involved left space for improvement. By introducing a different kernel function, so called Cauchy kernel, Mc Cormack and Sherstyuk [MS98] provided the first closed-form formulae for primitives based on line-segments, arcs of circle and triangles. The new profile of computational efficiency [She99] called for further investigation on the technique. Angelidis and Hornus [AC02, HAC03] introduced convolution surfaces of varying radius, using linear weight function along line-segments for power-inverse kernels of degree 2 and 3 , together with a subdivision strategy. In addition to providing similar formulae for the Cauchy kernel, Jin [JT02b, JT02a] extended the method to polynomial weight up to degree 3 over all quadratic skeleton curves. Recently, a recurrence formula was introduced in [HC11] to obtain closed-form primitives along linesegments for power inverse and generalized Cauchy kernels of arbitrary order, with polynomial weight of any degree.

An alternative solution for getting complex analytical implicit primitives, first used in [WO97], is to warp simpler implicit primitives through a transformation such as Barr's bend, twist or taper [Bar84]. As we shall see, these transformations do not preserve the circular quality of the normal cross-section of the primitive (see Figure 10, first row), and thus cannot be applied as is for our purpose.

\subsection{Overview}

Our work builds on the two approaches presented above: we introduce specific space warps to get artefactfree analytical implicit primitives along circular helices. The latter are based on closed-form convolution with varying radius over line segments or arcs of circles.

Our first contribution, described in Section 2, is the introduction of convolution primitives for arcs of circles with polynomial weights and families of infinite support kernel. A new piecewise defined warp then provides analytical expressions for helical implicit primitives.

Our second contribution, presented in Section 3, is the introduction of artefact-free space warps to generate helical primitives from line-segment ones.

We show in Section 4 that these two alternatives are complementary and provide methods for the whole range of helical skeletons. We additionally show that, for a lower computational cost, the shapes based on our helical primitives are visually more appealing than those obtained with a standard tessellation into line segments.
Notations: Without loss of generality we consider that the helical skeleton to be processed is given by:

$$
\left\{\begin{array}{l}
x=R \cos (u) \\
y=\epsilon R \sin (u) \\
z=S u
\end{array}\right.
$$

where $\epsilon= \pm 1$ and $u \in I \subset \mathbb{R} . I$ is the interval defining the part of the helix used as skeleton. The length of the latter is thus $\sqrt{R^{2}+S^{2}}|I|$. The twis is defined as the ratio $\frac{S}{R}$; we shall see that it dictates the warp to be used. When this ratio tends to 0 (respectively to $\infty$ ) the helix tends to a circle (respectively to a line segment). Figure 2 gives some examples of the shape of the helix according to the twist. The thickness of the primitive is assumed to be smaller than the radius of curvature of the helix, as otherwise the warps run into singularities.

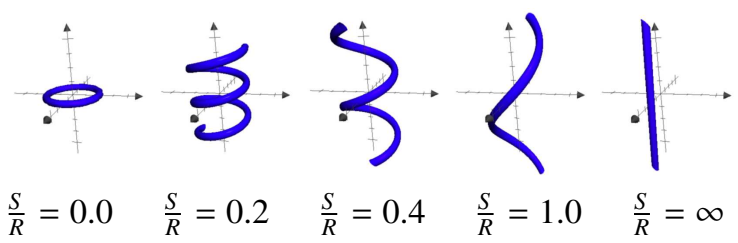

Figure 2: Shape of the helix for increasing value of $\frac{S}{R}$.

The Cartesian coordinates of a point $P$ in $3 \mathrm{D}$ space are noted $(x, y, z)$. Its cylindrical coordinates are $(r, \theta, z)$ where $\theta$ is the planar angle with the $x$-axis (see Fig. 3(a)).

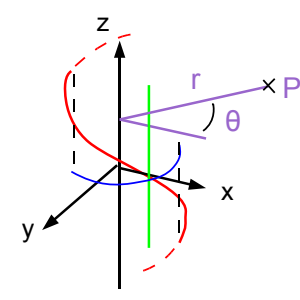

(a)

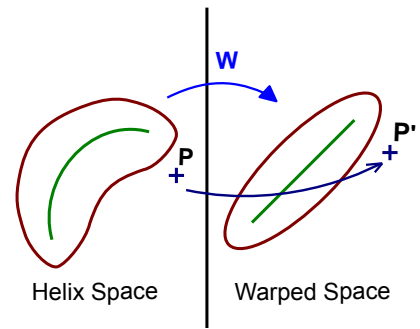

(b)
Figure 3: (a) The z-axis helix studied with the virtual segment (green) and virtual circle (blue) we use for warping. (b) Principle of a warping for implicit primitive as used in [WO97].

Our further derivations are applicable to common superset of Cauchy and power inverse kernels of arbitrary orders. Those laters are defined as:

$$
K(r)=\frac{1}{\left(1+s r^{2}\right)^{i / 2}} \text { and } K(r)=\frac{1}{r^{i}} .
$$

For a skeleton given by a parametrized curve $\Gamma$ : $[a, b] \rightarrow \mathbb{R}^{3}$ with a polynomial weight function $\mathfrak{g}$ the 
associated convolution function is given by

$$
f(P)=\int_{a}^{b} \mathfrak{g}(t) K(d(\Gamma(t), P))\left|\Gamma^{\prime}(t)\right| \mathrm{d} t .
$$

The warps we make use of are space transformation $W: \mathbb{R}^{3} \rightarrow \mathbb{R}^{3}$ that maps the circular helix to a simpler skeleton curve (see Fig. 3(b)). The helical primitive function $\tilde{f}$ we look for is then defined from the simpler skeleton primitive $f$ by:

$$
\tilde{f}(P)=f(W(P))
$$

\section{From circle to helical primitives}

In this section we first introduce closed form convolution primitives for arcs of circle with a polynomial weight, a case never solved so far. Our approach applies uniformly to both Cauchy and power inverse kernels of even order; we include as an example the closed form expression for a linear weight in the case of the classical (i.e. order 4) Cauchy kernel. We then present a warp that maps the helix onto a circle. This provides a first method for computing an analytical helical primitive.

\subsection{Closed-form primitive for arcs of circles}

When it comes to integration, rational functions are the dependable class [Bro05]. The main ingredient in obtaining closed-form convolution primitives for arcs of circle is to introduce an appropriate rational parameterization.

We assume that the points $O, A$ and $B$ (see Fig. 4) are not aligned and such that $|O A|=|O B|=r$. They define a plane in space and two arcs of the same circle, one of angle $\alpha$ the other of angle $\pi+\alpha$, where $0<\alpha<\pi$ is given by $\alpha=\arccos \left(\frac{\overrightarrow{O A} \cdot \overrightarrow{O B}}{r^{2}}\right)$. According to which of the two arcs we deal with $T=\tan \left(\frac{\alpha}{4}\right)$ or $T=$ $\tan \left(\frac{\pi+\alpha}{4}\right)$.

Momentarily we consider the coordinate system $(x, y, z)$ where the origin is the center of the circle, the $x$-axis is the bisector of the chosen angle defined by $O, A$ and $B$ and the $(x, y)$ plane is the plane of the circle. A parametrization of the arc of circle is then given by

$$
\begin{aligned}
\Gamma:[-T, T] & \rightarrow \mathbb{R}^{3} \\
t & \mapsto\left(r \frac{2 t}{t^{2}+1}, r \frac{t^{2}-1}{t^{2}+1}, 0\right) .
\end{aligned}
$$

When $t \rightarrow \pm \infty, \Gamma(t)$ tends to the point diametrically opposite to the mid-point of the arc. The other points of the circle are obtained for a parameter $t \in \mathbb{R}$. In terms of this parameter $t$ the infinitesimal arc length is $\left|\Gamma^{\prime}(t)\right|=\frac{2 r}{t^{2}+1}$.

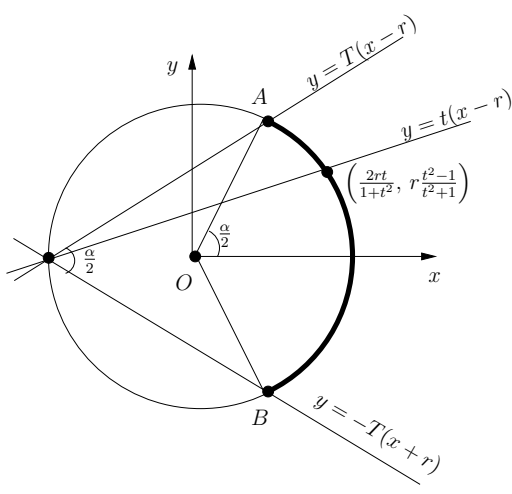

Figure 4: Parameterization of the arc of circle

Consider a point $P(x, y, z)$ in space. We have

$$
|P \Gamma(t)|^{2}=\frac{\alpha t^{2}-2 \beta t+\gamma}{t^{2}+1}
$$

where $\alpha=x^{2}+(y-r)^{2}+z^{2}, \beta=2 r x, \gamma=x^{2}+(y+r)^{2}+z^{2}$. Note that

$$
\begin{aligned}
\gamma+\alpha & =2\left(|O P|^{2}+r^{2}\right), \\
\alpha T^{2}+2 \beta T+\gamma & =\left(T^{2}+1\right)|A P|^{2}, \\
\gamma T^{2}-2 \beta T+\gamma & =\left(T^{2}+1\right)|B P|^{2},
\end{aligned}
$$

so that $(\alpha, \beta, \gamma)$ is actually the solution of a linear system that depends on $T$ and the squares of the distances of $P$ to $O, A$ and $B$. There is a unique solution provided that $A, O$ and $B$ are not aligned, i.e. $T\left(T^{2}-1\right) \neq 0$. An explicit expression for $(\alpha, \beta, \gamma)$ is obtained by Cramer's rules.

Hence the convolution primitive for the arc of circle with weight $\mathfrak{g}:[a, b] \rightarrow \mathbb{R}$ is given by:

$$
C_{O A B}(P)=2 r \int_{-T}^{T} \mathfrak{g}(t) \frac{\left(t^{2}+1\right)^{\frac{i}{2}-1}}{\left(a t^{2}-2 b t+c\right)^{\frac{i}{2}}} d t
$$

where $a=1+s \alpha, b=s \beta, c=1+s \gamma$ for Cauchy kernels and $a=\alpha, b=\beta, c=\gamma$ for power inverse kernels (of order $i$ ). Closed form expressions for the following integrals are given in [HC11].

$$
I_{k, i}=\int \frac{t^{k}}{\left(a t^{2}-2 b t+c\right)^{\frac{i}{2}}} d t
$$

as they appear in line-segment primitives. When using even order Cauchy or power inverse kernels those expressions also provide closed form primitives for arcs of circle with polynomial weight function $\mathfrak{g}$. It is just a matter of expanding the numerator. For odd order kernels, the closed form primitives call on elliptic functions. 

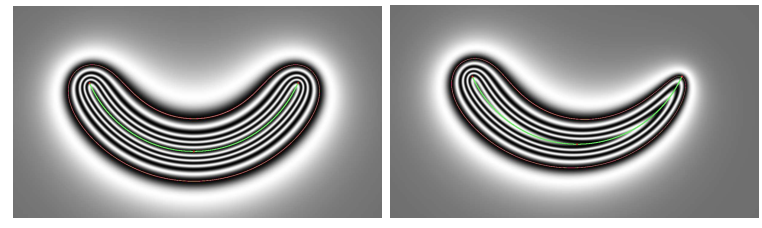

Figure 5: The level sets of the convolution primitive for an arc of circle for constant (right) and linear (left) weight.

Consider the particular case of an arc of circle with a linear weight defined by the two endpoints weights $\mathfrak{g}_{A}$ and $\mathfrak{g}_{B}$, that is: $\mathfrak{g}(t)=u t+v$ where $u=\frac{\mathfrak{g}_{A}-\mathfrak{g}_{B}}{2 T}, v=\frac{\mathfrak{g}_{B}+\mathfrak{g}_{A}}{2}$ so $C_{A O B}(P)$. For the classical (order 4 ) Cauchy kernel the primitive is given by

$2 r\left[u\left(I_{4,3}(a, b, c)+I_{4,1}(a, b, c)+v\left(I_{4,2}(a, b, c)+I_{4,0}(a, b, c)\right)\right]_{-T}^{T}\right.$.

The explicit expressions for $I_{4,0}, \ldots, I_{4,3}$ are given in [HC11]. The left hand sides of Equations 2-4 arise as subexpressions and it is worth using those latter for a faster evaluation. Figure 5 illustrates the level sets of this primitive in the case where $\mathfrak{g}_{A}=\mathfrak{g}_{B}$ and in the case where $\mathfrak{g}_{A} \neq \mathfrak{g}_{B}$.

\subsection{Warp based extension to helical primitives}

A first way to generate an analytical, convolution-like field along a helical skeleton is to use a warping method, based on a piece-wise map of the helix onto arcs of a circle. More precisely, we use a translation along the $z$-axis, where the amount of translation depends on the $\theta$ coordinate of the query point $P$ :

$$
W(r, \theta, z)=\left\{\begin{array}{c}
r \\
\theta \\
z-\tau(\theta)
\end{array}\right\} \text { with } \tau(\theta)=\epsilon S \theta
$$

For $\tau$ to be continuous, we use more than two arcs of circle for mapping a turn of the helix (using a different warp per arc of circle): this way, the warp is injective and $O, A, B$ are not aligned (see Section 2.1). More precisely, we define a warp of an arc of helix using $\tau(\theta)=\epsilon . S \theta$ on $\left[-\frac{3 \pi}{4}, \frac{3 \pi}{4}\right]$ which includes all the points of the helix arc. We use a cubic spline junction to have a continuous warp defined everywhere in space (see Fig. 6). Results are depicted on Figure 8.

\subsection{Handling of thick helical primitives}

The warp introduced in previous section is not well defined along the helix axis and bears no continuous extension. As we go across the axis, from $\theta$ to $\theta+\pi, \tau$ takes a non zero step unless $\theta=0, \pm \pi$. As a result the scalar field for the primitive is singular along the axis. Plotting its level sets in the vicinity of the axis is thus

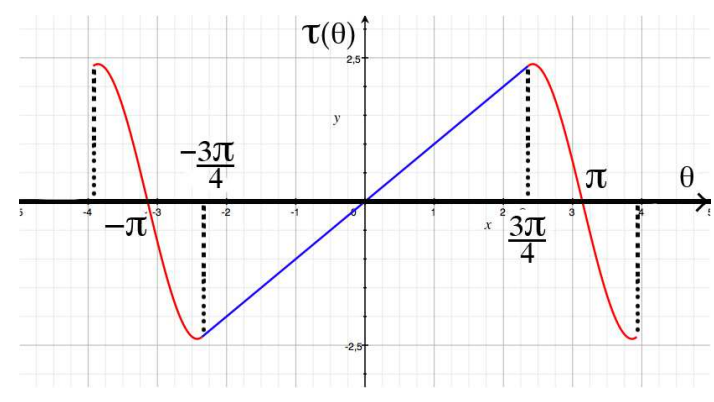

Figure 6: Amount of translation along the z-axis according to the angle $\theta$ in cylindrical coordinates

error prone. The situation is remedied by increasing the dimension of the problem: a fourth coordinate $t$ is introduced. This latter is made to tend to $\infty$ when $r$ tends to 0 and equals 0 when $r \geq R$. The initial circle primitive used for the definition of a convolution surface depends on three parameters, which are the distances between a point in space and the points $O, A$ and $B$. By introducing the parameter $t$, the circle used for the convolution is actually embedded in a 4 dimensional space. As shown in Figure 7, this allows us to remove the pinched area in the vicinity of the axis of the helix.
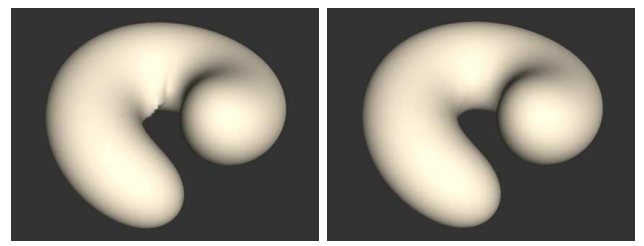

Figure 7: Right: for a thicker volume around the helix, a pinched blend occurs in the vicinity of the axis if we use the warp of Section 2.2. Left: the lift of Section 2.3 erases this unwanted singularity.

The improved warp allows us to generate implicit primitives for highly twisted helices. This is illustrated in Figure 8. For the top row we used a constant weight function on the circle. The helical primitive has then a constant radius as is expected. For the bottom row we used a linear weight function. That reflects in a varying radius along the helix that we can wish for.

The deformation introduced by the above circle based warp on the normal cross-section to the helix becomes noticeable as $\frac{S}{R}$ increases, i.e. when the helix becomes closer to a line-segment. Typically, when $\frac{S}{R}$ is greater than 0.6 the ratio between the incircle and excircle of the section becomes smaller than 0.8 . We thus offer next a helical primitive using a warp onto a line-segment. Section 4 shows how to combine them to adequately span the full range of circular helices. 


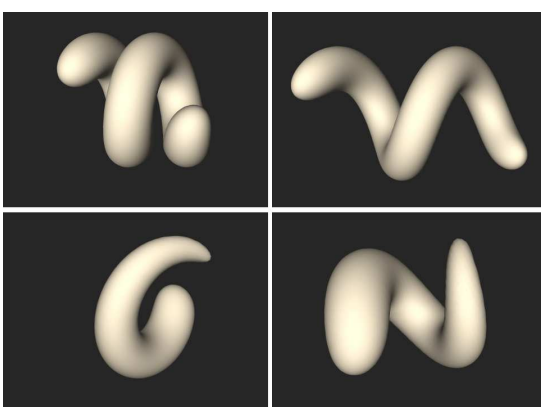

Figure 8: Helical primitives based on warps to the circle. Top: constant weight. Bottom: varying weight.

\section{Helical primitives by warping to a line segment}

In this section we shall introduce warps of a helix to a line segment providing helical primitives of increasing quality. Our starting point is the inverse of Barr's twist, which was already used in [WO97] to create implicit primitives. We exhibit the imperfections of this warp for our purpose and offer new transformations to compose it with to obtain the desired results. Those new warps form an essential part of our contribution in this article.

As a convention for the rest of this section we use the line segment parallel to the axis of the helix and shown in green in Figure 3(a). In contrast to the method of previous section, any number of turns in the helix can be handled in a single warp.

\subsection{Naive twist-based warps}

Twisting back a helix of parameter $S$ to a linesegment can be done by setting $v$ to $-\frac{\epsilon}{S}$ in the equation of a twist:

$$
\operatorname{twist}(v,(x, y, z))=\left\{\begin{array}{c}
x \cos (v \cdot z)-y \sin (v \cdot z) \\
x \sin (v \cdot z)+y \cos (v \cdot z) \\
z
\end{array}\right\}
$$

We therefore define our basic warp as:

$$
W_{t w i s t}(x, y, z)=t w i s t\left(-\frac{\epsilon}{S},(x, y, z)\right)
$$

As shown in the top row of Figure 10, the normal sections of the resulting primitives are not circular. To get some insight we consider a helix with $R=1, \epsilon=1$ and examine the linear part, $W_{\text {simple }}$, of the map $W_{\text {twist }}$ around $(1,0,0)$ :

$$
W_{\text {simple }}(x, y, z)=\left\{\begin{array}{c}
x \\
y-\frac{z}{S} \\
z
\end{array}\right\} .
$$

The preimage of the vector $(0,0,1)$ is the tangent vector to the helix at the point $(1,0,0)$, that is $(0,1 / S, 1)$. Figure 9(a) depicts a circular cylinder, the axis of which is given by this tangent vector, and its image under $W_{\text {simple }}$. This latter is a cylinder with an elliptic section. Indeed the intersection of the left hand side cylinder with a plane $z=c$ is an ellipse $\mathcal{E}$ and the restriction of $W_{\text {simple }}$ to that plane is a translation within this plane. Therefore the section of the image cylinder is the same ellipse $\mathcal{E}$. As explained on Figure 9(b) the ratio of the major and minor radius of this ellipse is:

$$
\frac{r_{\min }}{r_{\max }}=\sin (\alpha)=\frac{S}{\sqrt{R^{2}+S^{2}}}
$$

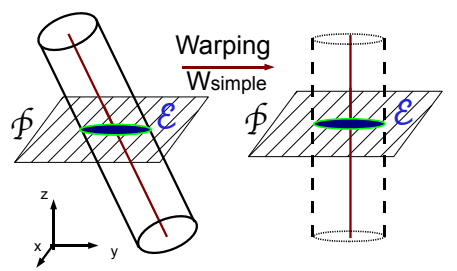

(a)

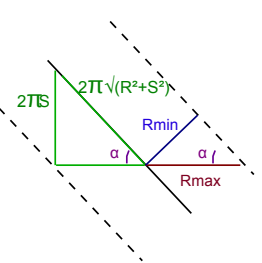

(b)
Figure 9: (a) Transformation $W_{\text {simple }}$ applied to an inclined cylinder, (b) Lateral view of that cylinder

Scaling the warp along the $y$-axis will bring the horizontal section closer to a circle. The resulting transformation is:

$$
\tilde{W}(x, y, z)=\left\{\begin{array}{c}
x \cos \left(\frac{\epsilon}{S} z\right)-y \sin \left(\frac{\epsilon}{S} z\right) \\
\frac{S}{\sqrt{R^{2}+S^{2}}}\left(x \sin \left(\frac{\epsilon}{S} z\right)+y \cos \left(\frac{\epsilon}{S} z\right)\right) \\
z
\end{array}\right\}
$$

However, this transformation still does not work for highly twisted helices: our additional scaling component neglected the effect of the rotation (if we take again the analogy with an oblique cylinder, the latter should rotate along the helix). It is more appropriate to apply the scaling correction to the angle in the cylindrical coordinates: $\bar{W}(r, \theta, z)=\left\{r, \frac{S}{\sqrt{R^{2}+S^{2}}}\left(\theta-\frac{\epsilon}{S} z\right), z\right\}$. This is equivalent to applying a rotation around the helix axis after the twisting. The angle of rotation depends on the polar coordinate after twisting. However the rotation to be considered for a helix is a rotation around its center of curvature.

\subsection{Correctly handling the central part}

The warps we described so far gave us some insight on the corrections required to get a reasonably circular cross-section: a scaling should be applied after a rotation around the local center of curvature of the helix. 


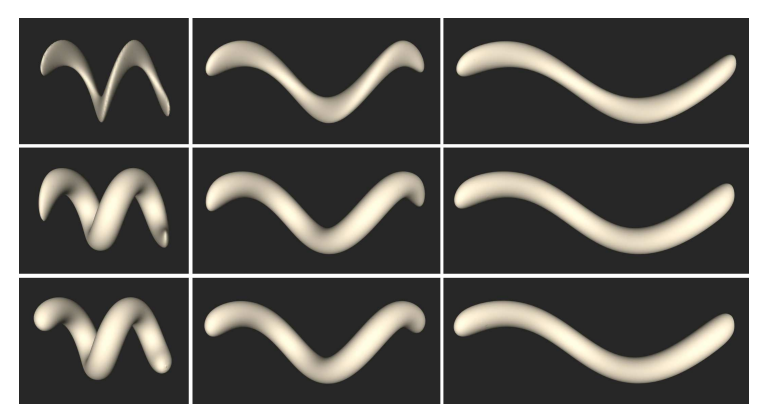

Figure 10: Comparison, for different values of $\frac{S}{R}$, between the classic twist (first row), the solution with the end point problem (second row) and our final solution (third row).

The center of curvature at a parameter $t$ along the helix is given by:

$$
\left(-\frac{S^{2}}{R} \cos (t),-\frac{S^{2}}{R} \sin (t), S t\right)
$$

When the helix tends to a line segment the centers of curvature wander off to infinity whereas, when the helix tends to a circle, the centers of curvature are attracted toward the axis of the helix. The correction we define thus varies smoothly from the value of $\tilde{W}$ to that of $\bar{W}$ according to the amount of twist of the helix.

Our new transformation can be written as:

$$
W(x, y, z)=g\left(\text { twist }\left(-\frac{\epsilon}{S},(x, y, z)\right)\right)
$$

where

$$
\begin{gathered}
g(x, y, z)=\left\{\begin{array}{c}
x \cos (\mu \psi(x, y, z))-y \sin (\mu \psi(x, y, z)) \\
x \sin (\mu \psi(x, y, z))+y \cos (\mu \psi(x, y, z)) \\
\frac{\sqrt{R^{2}+S^{2}}}{S} z
\end{array}\right\},(6) \\
\mu=\frac{S}{\sqrt{R^{2}+S^{2}}}-1,
\end{gathered}
$$

and

$$
\psi(x, y, z)=\operatorname{atan} 2\left(y, x+\frac{S^{2}}{R}\right) .
$$

The restriction of $g$ to a plane orthogonal at some point to the helix axis is a rotation around the center of curvature of the helix at this point. This is equivalent to multiplying the polar coordinates, with respect to the center of curvature, of the point by the ratio $\frac{S}{\sqrt{R^{2}+S^{2}}}$ (Equation 5). Another important feature is to ensure that the helical primitive has the same thickness as the associated line segment primitive. The length of the skeleton needs to be preserved to ensure this. As the last coordinate of $g$ in Equation 6 discloses it, this is obtained by scaling along the z-axis.
The primitives obtained with this new warp are illustrated in the second row of Figure 10. To obtain a helical primitive with varying radius, we can simply assign a polynomial weight function on the line-segment skeleton the helix is warped onto and use the results of [HC11]. Results are shown in Figure 11.

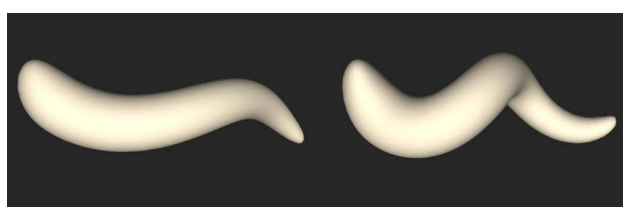

Figure 11: Helical primitives with varying radius.

\subsection{Final solution for segment-based primitives}

Figure 10 provides a comparison between the results obtained with the original twist and those obtained with the presented new warp. A closer look at the leftmost image in the second row reveals an unwanted artefact toward the end points of the shape. This artefact furthermore prevents the seamless blends that are sought when the helix is part of a more complex skeleton.

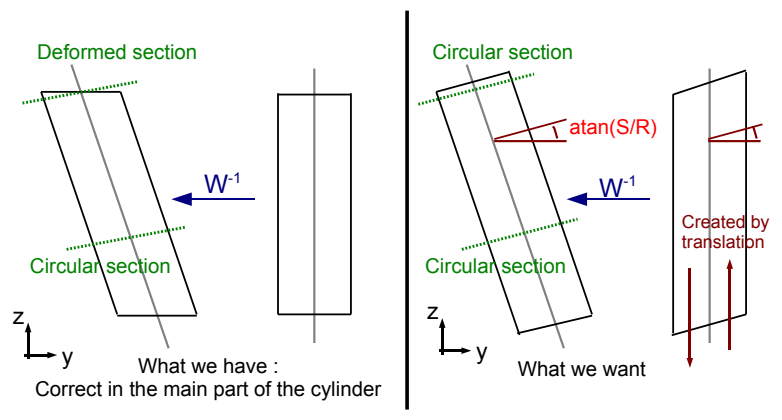

Figure 12: Idea justifying the correction of both ends of the primitive.

The artefacts observed around the tips of the helical primitive can be explained through the linearized warp $W_{\text {simple }}$ already studied in Section 3.1. Figure 12 illustrates the cause of the problem at the end points and how to measure the unwanted deformation in order to introduce an appropriate correction. A translation along the $z$-axis, the norm of which depends linearly on $y$ in the deformed space, is enough to correct the tips in this linearized version. As before, this correction is adapted to our main transformation through a translation that depends on the polar coordinate with respect to the center of curvature. This is given explicitly by:

$$
W_{\text {correct }}(x, y, z)=\tau(W(x, y, z))
$$


where

$$
\tau(x, y, z)=\left\{\begin{array}{c}
x \\
y \\
z-\epsilon \frac{R^{2}+S^{2}}{S} \psi(x, y, z)
\end{array}\right\}
$$

The map $\tau$ introduces a discontinuity of the field function when $\psi= \pm \pi$. The latter can be easily overcome through a linear or spline junction as was done for warps onto arcs of circles (Figure 6).

The improvement introduced by this last adjustment can be easily appreciated by comparing the second and third row in Figure 10.

\section{Evaluation and combination of our solutions}

We presented two ways of obtaining a helical primitive by warping the helix either on arcs of a circle or onto a line segment. We lead an experimental evaluation of the results obtained through those two warps. On one hand, the warp to circle solution presented in Section 2 leads to good results when $\frac{S}{R} \in[0, \approx 0.5]$ (see Figure 2 to have an idea of the shape of the helix). Beyond that the shape of the cross-section is noticeably deformed. We may think of improving the situation by introducing corrections as was done for line segments in Section 3. On the other hand, the solution based on warp to line segment developed in Section 3 gives good results for all kinds of helices, except when the helix becomes highly twisted: for a helix close to a circle, our solution still locally flattens the primitive around the end points of the helix. In our experiments, this is negligible until $\frac{S}{R}$ becomes lower than $\approx 0.35$ (instead of $\approx 0.7$ without the final correction for the tips). Thus, the whole range of helices can be handled nicely by at least one of the two methods we proposed, with good results.

\section{Combination of the two methods}

Since the quality of the result depends on the same ratio $\frac{S}{R}$, automatically choosing the method to apply is very easy.

A first solution is to use a segment based primitive, which is computationally cheaper, when $\frac{S}{R}>0.4$, a circle based primitive when $\frac{S}{R}<0.3$ and an intermediate solution in-between. This intermediate solution is constructed by interpolation, for instance a cubic interpolation. In this first approach, the evaluation of the helical primitive when $0.3<\frac{S}{R}<0.4$ requires both the evaluation of the circle based primitive and the line segment based primitive. A more efficient way to handle the transition zone of twisting, i.e. when $0.3<\frac{S}{R}<0.4$, is to work with a warp of a line segment for the middle part of the helix and only two warps onto arcs of circles for the tips of the shape. Then interpolation between the two different primitives occurs only within a limited range of the $\mathrm{z}$ coordinate (see figure 13).

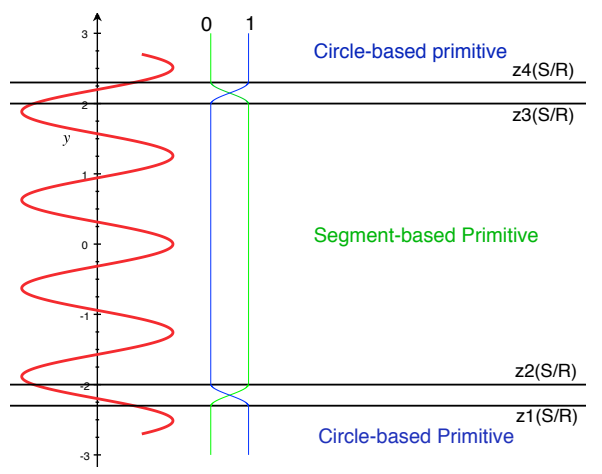

Figure 13: Transition between the two kind of primitives in function of $\frac{S}{R}$

Figure 14 emphasizes the quality of our global helical primitive in the context of a more complex skeleton. The helix is prolonged by a line segment with continuous tangency. The resulting shape remains smooth around the transition between the helical primitive and the line segment primitive.
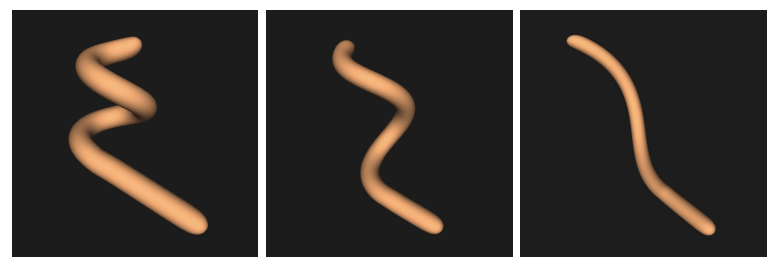

Figure 14: Helical primitive prolonged by a segment primitive for different values of $\frac{S}{R}$

\section{Computational efficiency}

\begin{tabular}{|c|c|c|c|}
\hline & $\begin{array}{c}\text { segment } \\
\text { primitive }\end{array}$ & $\begin{array}{c}\text { helical } \\
\text { from circle }\end{array}$ & $\begin{array}{c}\text { helical } \\
\text { from segment }\end{array}$ \\
\hline $\begin{array}{c}\text { Time for } 200^{3} \\
\text { field queries }\end{array}$ & $1.45 \mathrm{~s}$ & $2.63 \mathrm{~s}$ & $3.27 \mathrm{~s}$ \\
\hline
\end{tabular}

Table 15: Computation time for a single primitive. CPU: $2.4 \mathrm{GHz}$ Intel Core 2 Duo (only one core used).

Table 15 compares the timings for the evaluations of our two analytical helical primitives and of a linesegment primitive. This shows that when 3 segments or more are needed to approximate a helical skeleton, our new helical primitive is much more efficient. The fact that the warp-to-circle solution used for highly twisted helices requires two primitives per turn does not change 


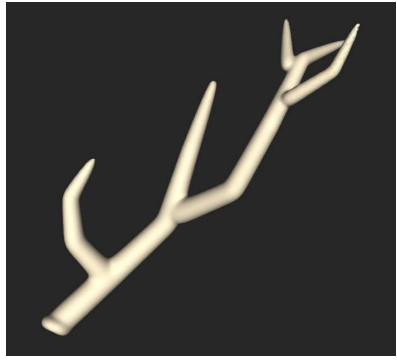

(a)

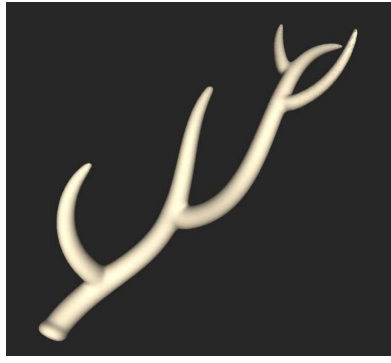

(b)

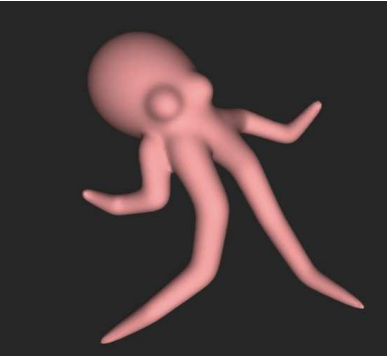

(c)

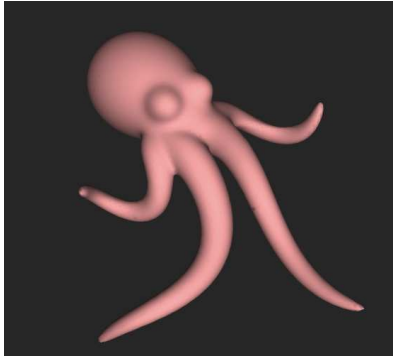

(d)

Figure 16: Comparison of model quality using a tessellation of 3D skeleton curves into line-segments (left) compared to our helical primitives (right) when a given computational time is allowed.

this result, since more than 6 segments per turn would be needed then. To make this comparison more visual, Figure 16 depicts the quality we would get, at constant computational cost, by tessellating a skeleton into linesegments or into helices. For this level of zoom, getting the same visual quality with a tessellation into line segments would respectively multiply computational time by factors 2.71 for the octopus, which includes non helical parts, and 5.84 for the antlers, which is only made of helices.

\section{Conclusion and Future work}

We have introduced the first analytical formulation for helical implicit primitives. The method handles primitives of varying radius and can be used with arbitrary convolution kernels such as power inverse and Cauchy kernels of arbitrary order. Indeed, a side, yet important, contribution is a new general formulation for arc of circle convolution primitives, which allows for varying radius.

Our solution for helices can be considered as a pseudo-convolution since it built on arcs of circle and line-segment convolution primitives by warping. Similarly to convolution, the resulting helical primitives can be used in complex configurations where the skeleton is tessellated into several helices: as with direct closedform convolution, the resulting curve is bulge-free.

We furthermore have set up the bases of a methodology to design advanced space warps that progressively build complex primitives from simpler ones. That opens the door to further extensions: the same methodology could be applied to get implicit primitives along $3 D$ spiral skeletons, which were shown to be very useful to represent a larger variety of natural shapes such as seashells and horns [HT11]. Modifying our deformation to the sweeping of more general cross-sections around the helix skeleton would also be a useful exten- sion of this work, made possible by our section preserving warp.

\section{References}

[AC02] Angelidis A., CANI M.-P.: Adaptive implicit modeling using subdivision curves and surfaces as skeletons. In 7th ACM Symposium on Solid Modeling and Applications, June, 2002 (Saarbrucken, Allemagne, June 2002), pp. 4552.

[BAC*06] Bertails F., Audoly B., Cani M.-P., Querleux B., Leroy F., LÉVÊQUE J.-L.: Super-helices for predicting the dynamics of natural hair. ACM Trans. Graph. 25, 3 (July 2006), 1180-1187. Special issue: SIGGRAPH'06.

[Bar84] Barr A. H.: Global and local deformations of solid primitives. Special issue: SIGGRAPH84 - Comput. Graph. 18 (January 1984), 21-30.

[Bro05] BronsteIn M.: Symbolic integration. I, 2nd Edition. Vol. 1 of Algorithms and Computation in Mathematics (2005).

[BS91] Bloomenthal J., Shoemake K.: Convolution surfaces. In Proceedings SIGGRAPH '91 (New York, NY, USA, 1991), ACM, pp. 251-256.

[HAC03] Hornus S., Angelidis A., CANi M.-P.: Implicit modelling using subdivision curves. Visual Comput. 19, 2-3 (May 2003), 94-104.

[HC11] Hubert E., Cani M.-P.: Convolution surfaces based on polygonal curve skeletons. Journal of Symbolic Computation (2011). http://hal.inria.fr/inria-00429358.

[HT11] Harary G., Tal A.: The natural 3D spiral. Computer Graphics Forum (Proceedings of Eurographics) 30, 2 (2011)

[JT02a] JIN X., TAI C.-L.: Analytical methods for polynomial weighted convolution surfaces with various kernels. Computer Graphics 26, 3 (2002), 437-447.

[JT02b] JIN X., TAI C.-L.: Convolution surfaces for arcs and quadratic curves with a varying kernel. The Visual Computer 18, 8 (2002), 530-546.

[MS98] McCormack J., Sherstyuk A.: Creating and rendering convolution surfaces. Computer Graphics Forum 17, 2 (1998), 113-121.

[She99] SHERsTyuk A.: Kernel functions in convolution surfaces: A comparative analysis. The Visual Computer 15, 4 (1999), 171-182.

[WO97] Wyvill B., Overveld K. V.: Warping as a modelling tool for csg/implicit models. In Proceedings of the 1997 International Conference on Shape Modeling and Applications (SMI '97) (Washington, DC, USA, 1997), IEEE Computer Society, pp. 205-214. 